\title{
PROPÁGULOS FÚNGICOS Y PARTÍCULAS CONTAMINANTES PRESENTES EN FOSAS NASALES DE VOLUNTARIOS EN LA UNIVERSIDAD AUTÓNOMA METROPOLITANA UNIDAD XOCHIMILCO
}

Fungal propagules and contaminant particles present in nostrils of volunteers in Universidad Autónoma Metropolitana Unidad Xochimilco

\section{Arturo MIRANDA-CALIXTO ${ }^{1}$, Judith CASTELLANOS-MOGUEL ${ }^{1 *}$ y Raúl Venancio DÍAZ-GODOY ${ }^{2}$}

${ }^{1}$ Laboratorio de Micología, Departamento El Hombre y su Ambiente, Universidad Autónoma Metropolitana Xochimilco, Calzada del Hueso 1100, Col. Villa Quietud, C.P. 04950, Tlalpan, Ciudad de México, México

${ }^{2}$ Departamento de Aceleradores, Instituto Nacional de Investigaciones Nucleares, Carretera México-Toluca-La Marquesa s/n, C.P. 52750, Ocoyoacac, Estado de México, México

*Autor para correspondencia: mjmoguel@correo.xoc.uam.mx

(Recibido: junio 2018; aceptado: enero 2020)

Palabras clave: bioaerosoles, hongos, lavados nasales, química elemental

\section{RESUMEN}

La emisión de contaminantes a la atmósfera de la Ciudad de México y zona metropolitana ha provocado problemas de calidad de aire. Parte de los compuestos inorgánicos provienen de procesos naturales y antropogénicos. Sin embargo, también están presentes bioaerosoles, como los hongos, capaces de provocar enfermedades respiratorias. El objetivo de la investigación fue un estudio preliminar para la recuperación e identificación de propágulos fúngicos a partir de muestras de miembros de la comunidad de la Universidad Autónoma Metropolitana -Xochimilco. A trece voluntarios se les realizaron lavados nasales al llegar a la universidad y después de una hora, se colocaron cajas de Petri para sedimentación por gravedad en los sitios donde estuvieron entre muestreos. Una parte de la muestra se sembró en medio agar rosa Bengala, otra fracción se observó en microscopio y otra se empleó para el análisis de química elemental. Como resultados los géneros fúngicos más frecuentes fueron Penicillium y Cladosporium, por sedimentación Penicillium. También se aislaron levaduras, las especies con mayor número de colonias en lavados nasales fueron Candida guilliermondii y C. tropicalis, por sedimentación Cryptococcus humicola. Dentro de los elementos identificados se encontraron trazas de metales como Ti, Zn, Cr, Fe y Ni. Los lavados nasales pueden ser empleados como una estrategia para conocer la microbiota fúngica en vías respiratorias, así como parte de estrategias preventivas dentro del diagnóstico de enfermedades respiratorias.

Key words: bioaerosols, fungi, nasal lavage, elemental chemistry

\begin{abstract}
The emission of pollutants into the atmosphere of Mexico City and the metropolitan area causes air quality problems. Part of the inorganic compounds comes from natural and anthropogenic processes. However, bioaerosols such as fungi are also present,
\end{abstract}


and they are capable of causing respiratory diseases. The objective of the investigation was a preliminary study for the recovery and identification of fungal propagules from samples of members of the Universidad Autonoma Metropolitana-Xochimilco community. Thirteen volunteers underwent nasal washes upon arrival at the university and after an hour, Petri dishes were placed for sedimentation by gravity in the places where they were between samplings. Part of the sample was placed in Bengal rose agar, another fraction was observed under a microscope and another was used for the analysis of elemental chemistry. As results, the most frequent fungal genera were Penicillium and Cladosporium, and Penicillium by sedimentation. Yeasts were also isolated; the species with the highest number of colonies from nasal washes were Candida guilliermondii and C. tropicalis, by sedimentation Cryptococcus humicola. Among the elements identified were traces of metals such as Ti, Zn, Cr, Fe, and Ni. Nasal washes can be used as a strategy to know the fungal microbiota in the respiratory tract, as well as part of preventive strategies in the diagnosis of respiratory diseases.

\section{INTRODUCCIÓN}

La contaminación atmosférica en el Valle de México y su zona metropolitana en gran parte es provocada por los contaminantes que se emiten a la atmósfera, las condiciones fisiográficas y el incremento demográfico. Los contaminantes del aire se pueden clasificar según su origen como naturales (erupciones volcánicas que producen emisiones de gases, vapores, polvos y aerosoles) o antrópicos (vehículos, industria, hogares, actividades comerciales y de servicios) (Escobedo et al. 2001, SEMARNAT 2011). Como parte de los contaminantes se encuentran el monóxido de carbono, el bióxido de carbono y los óxidos de azufre y de nitrógeno, vapores de hidrocarburos, clorofluorocarbonos y los metales pesados (Escobedo et al. 2001). Su presencia provoca el deterioro de la calidad del aire e implica un problema latente, principalmente para la salud de la población (SEMARNAT 2011).

Los aerosoles son partículas suspendidas en la atmósfera y cuentan con un diámetro menor a 100 $\mu \mathrm{m}$. Estas partículas se encuentran como polvos, humos, neblinas y emanaciones, son resultado de la combustión, de la condensación de gases o vapores y de reacciones químicas (Escobedo et al. 2001). En México existen instancias como la Red Automática de Monitoreo Atmosférico (RAMA), cuyo objetivo es proporcionar información sobre la calidad del aire en forma sistemática y permanente $y$, en relación con los factores meteorológicos y urbanos, permite detectar la presencia y tendencia de contaminantes (Ramírez 2000). Sin embargo, también se encuentran suspendidos en el aire los bioaerosoles, partículas de procedencia biológica de tamaño microscópico, que pueden estar constituidos por virus, bacterias, propágulos fúngicos, polen, amebas y en general cualquier resto de microorganismo con un diámetro aerodinámico comprendido entre $100 \mu \mathrm{m}$ y $0.5 \mu \mathrm{m}$ (Cox y Wathers 1995).

Los bioaerosoles fúngicos constituyen la mayor parte de las partículas de origen biológico liberadas a la atmósfera (Hinds 1999, Yeo y Kim 2002, Wu et al. 2004). Distintos estudios han reportado que la presencia en la atmósfera de propágulos fúngicos es relevante para la ocurrencia de problemas de salud pública (Dutkiewicz 1997, Verhoeff y Burge 1997, Cooley et al. 1998, Bush y Portnoy 2001). Diversas investigaciones han descrito la presencia de material fúngico en interiores (Rosas et al. 1997, Codina et al. 2008) y exteriores (Rosas et al. 1986, 1990, Gioulekas et al. 2004, Oliveira et al. 2010). La presencia de estos bioaerosoles tiene una gran importancia, particularmente en áreas de trabajo y el hogar (Verhoeff y Burge 1997), donde pueden causar complicaciones en infecciones, sobre todo a pacientes inmunodeprimidos (Denning et al. 1997).

Algunas de las reacciones más comunes causadas por las partículas biológicas son de tipo alérgico, en la piel o en el tracto respiratorio, erupciones, fiebre alta, asma y escurrimiento nasal. Las principales vías de exposición a estos propágulos son por inhalación, ingestión y contacto con la piel. La inhalación es la que da lugar a los mayores problemas para la salud (Hafidh et al. 2007, Rodríguez et al. 2008). Dentro del amplio intervalo de tamaños de los bioaerosoles, los conidios fúngicos registran diámetros entre $1 \mu \mathrm{m}$ a $30 \mu \mathrm{m}$ (Reponen et al. 2001). Desde el punto de vista sanitario, los que tienen un tamaño menor o igual a $10 \mu \mathrm{m}$, pueden ser inhalados y los más pequeños pueden depositarse a nivel alveolar, lo que en algunos casos puede provocar reacciones alérgicas e infecciones (de la Rosa et al. 2002). 
En la nariz, los propágulos pueden ser retenidos por barreras físicas como la mucosa (Kacker 2010). Entre las pocas investigaciones sobre la microbiota fúngica nasal están la de Sercombe et al. (2006), que reportan la presencia de los géneros Alternaria, Cladosporium, Epicoccum, Penicillium, además de levaduras y la de Hafidh et al. (2007), quienes identificaron Penicillium, Candida albicans, Aspergillus flavus, Candida parapsilosis, Alternaria, Paecilomyces variotii, A. niger y C. krusei.

Existe un interés creciente en el entendimiento del rol que juegan los hongos aerotransportados en las enfermedades respiratorias (Torres-Rodríguez et al. 2012). Por tal motivo, el objetivo del presente trabajo fue la recuperación e identificación de propágulos fúngicos a partir de lavados nasales en trece voluntarios y determinar la composición de química elemental de las partículas presentes en las muestras, como estudio preliminar para conocer la composición de la microbiota fúngica en la población de la Ciudad de México.

\section{MATERIALES Y MÉTODOS}

\section{Voluntarios}

A trece alumnos de la Universidad Autónoma Metropolitana Unidad Xochimilco, voluntarios para el estudio, se les aplicaron lavados nasales. A los participantes se les informaron los objetivos del estudio, respondieron un cuestionario con información básica sobre sus hábitos, antecedentes de enfermedades respiratorias y dieron su consentimiento escrito para satisfacer las normas del Comité de Ética de Investigación de la propia Universidad. Los protocolos se diseñaron de acuerdo con lo establecido en la Declaración de Helsinki de la Asociación Médica Mundial de 1975 (WMA 1964), la NOM087-SEMARNAT-SSA1-2002 (SEMARNAT 2002) y el Manual de Bioseguridad en el Laboratorio de la Organización Mundial de la Salud (OMS 2005).

\section{Lavados nasales}

Para obtener las muestras provenientes de los voluntarios, se les realizaron lavados nasales en ambas fosas con un proceso modificado del de Sercombe et al. (2006) y del de Hafidh et al. (2007). Se emplearon $5 \mathrm{~mL}$ de agua desionizada y esterilizada en cada fosa nasal. Con una jeringa sin aguja se introdujeron los 5 $\mathrm{mL}$ y se retuvieron durante un minuto, posteriormente se utilizaron tubos viales estériles para recuperar el líquido. Los lavados nasales se realizaron en el Laboratorio de Micología del Departamento El Hombre y su Ambiente, la primera muestra se tomó en el momento que los alumnos llegaron a la universidad y una hora después se les realizó un segundo lavado.

Una vez obtenidas las muestras, se agregaron $50 \mu \mathrm{L}$ de cada una a cajas de Petri con agar rosa de Bengala (ARB) DIFCO ${ }^{\mathrm{TM}}$ con $500 \mathrm{mg}$ de cloranfenicol/L, se extendió la alícuota con un rastrillo y se incubaron a $28^{\circ} \mathrm{C}$ durante siete días. Al líquido restante de los lavados nasales se le agregó glutaraldehido al $50 \%$ para evitar la germinación de conidios y favorecer la conservación de los mismos en refrigeración.

\section{Sedimentación por gravedad en cajas de Petri}

De acuerdo con el sitio donde se encontraban los voluntarios durante el periodo entre lavados nasales, se colocaron cajas de Petri con ARB adicionado con cloranfenicol durante 15 minutos, con la finalidad de comparar los géneros cultivables obtenidos a partir de cada alumno y los presentes en el aire. Se colocaron las cajas en cinco zonas. De estas, tres en exteriores $\mathrm{y}$ dos en interiores.

\section{Conteo de unidades formadoras de colonias e identificación de géneros fúngicos}

Al paso de los siete días de incubación, se cuantificaron las unidades formadoras de colonias (UFC) y se observaron y registraron las características macromorfológicas de cada una de ellas. Posteriormente se procedió a realizar la identificación micromorfológica a nivel de género, que se llevó a cabo al colocar una muestra de cada colonia en portaobjetos con tinción Azul de Lactofenol para su observación en microscopio óptico. Este proceso se realizó con base en Barron (1968), Barnett y Hunter (1972) y Von Arx (1981). Por su frecuencia de aparición en las muestras, los hongos aislados se clasificaron según la escala de Yadav y Madelin (Esquivel et al. 2003), los valores se representan en el cuadro I.

\section{Levaduras}

Las levaduras obtenidas por lavados nasales y sedimentación por gravedad fueron resembradas por separado para obtener cultivos axénicos, necesarios para llevar a cabo la identificación a nivel especie con el sistema $\mathrm{API}^{\circledR} 20 \mathrm{C}$ AUX. Esta galería está constituida por cúpulas que contienen substratos deshidratados y permiten efectuar 19 ensayos de asimilación. Las cúpulas se inoculan con un medio mínimo semi agar y las levaduras crecen solamente si son capaces de utilizar el sustrato. Las galerías se colocaron en una incubadora con temperatura de $28^{\circ} \mathrm{C}$ a $30^{\circ} \mathrm{C}$, y se anotaron los resultados a las 48 y 72 horas. La información obtenida se analizó con el programa APIWEB $^{\circledR}$. 
CUADRO I. CRITERIOS DE CLASIFICACIÓN DE YADAV Y MADELIN, UTILIZADOS PARA MEDIR LA FRECUENCIA DE APARICIÓN DE LOS GÉNEROS FÚNGICOS

\begin{tabular}{cc}
\hline \multicolumn{2}{c}{ Clasificación de Yadav y Medelin } \\
\hline Categoría & Frecuencia de aparición \\
\hline Muy común & $80-100 \%$ \\
Común & $61-80 \%$ \\
Frecuente & $41-60 \%$ \\
Ocasional & $21-40 \%$ \\
Raro & $0.1-20 \%$ \\
No encontrados & \\
\hline
\end{tabular}

\section{Observación de muestras líquidas al microscopio}

De cada muestra de lavados nasales se tomaron $100 \mu \mathrm{L}$, se repartieron en 10 portaobjetos y se observaron al microscopio. Los resultados obtenidos se consideraron los contaminantes totales con los que los voluntarios tuvieron contacto. La morfología de las partículas encontradas fue comparada con la descrita en el manual de Lacey y West (2006).

\section{Composición química elemental}

Para este proceso se seleccionaron siete muestras líquidas que fueron transportadas al Instituto Nacional de Investigaciones Nucleares, donde fue necesario lavar la pared interna de los viales con $500 \mu \mathrm{L}$ de agua bidestilada para que el material que pudiera estar adherido escurriera. El contenido se depositó en tubos Eppendorf y se centrifugaron durante 15 min a $10000 \mathrm{rpm}$. Se extrajo una porción de líquido y volvió a centrifugarse durante 5 min para concentrar las partículas, al finalizar se extrajo el botón, se dejó secar y posteriormente se pulverizó. El material resultante se montó en cintas de carbono y se les agregó un recubrimiento de oro para su análisis por microscopía electrónica de barrido.

\section{RESULTADOS}

\section{Identificación de géneros fúngicos y levaduras}

Se identificó un total de veinticuatro géneros de hongos filamentosos y once especies de levaduras provenientes de los voluntarios y del ambiente. En los lavados nasales, los hongos filamentosos con mayor frecuencia de aparición fueron Penicillium y Cladosporium, mientras que en el ambiente universitario Pencillium. En cuanto a las levaduras, las de mayor frecuencia fueron Candida guilliermondii y Candida tropicalis en voluntarios y Cryptococcus humicola en las muestras de sedimentación por gravedad (Cuadro II). De las cajas sembradas con lavados nasales, no se observó crecimiento de micelio en el $52.307 \%$.

\section{Contaminantes totales en muestras líquidas}

El cuadro III presenta los contaminantes encontrados en las muestras obtenidas de lavados nasales, así como el número de muestras en las que fueron observados, porcentajes por muestra, total de registros y porcentaje total. En el caso de algunas esporas, es muy característica su morfología, por lo que se consideraron tipo Alternaria, tipo Fusarium y del grupo Aspergillus/Penicillium. También se encontraron restos de artrópodos, partículas de combustión, fragmentos vegetales y fibras probablemente provenientes de ropa, entre otros.

\section{Química elemental}

El análisis de química elemental por microscopía electrónica de barrido permitió detectar trazas de elementos como C, O, Ca, Ti, Na, Mg, Al, Si, P, K, $\mathrm{Cr}, \mathrm{Fe}, \mathrm{Ni}$ y $\mathrm{Cl}$. La figura 1 muestra los elementos detectados en cuatro muestras.

\section{DISCUSIÓN}

En México existe poca documentación sobre la presencia de propágulos fúngicos suspendidos en el aire, Entre los géneros dominantes reportados tanto en interiores como en exteriores se encuentran Cladosporium, Alternaria, Penicillium, Aspergillus, Coprinus, Curvularia, Venturia, Fusarium, Acremonium, Bipolaris, Geotrichum, Helminthosporium y Monila (Rosas et al. 1997, Sáenz y Gutiérrez 2003, Ponce-Caballero et al. 2010, Rocha et al. 2013, Rosique et al. 2013, Hernández-Castillo et al. 2014). En México sólo existe un reporte (Rodríguez-Orozco et al. 2008) de identificación de hongos obtenidos por exudados nasales y faríngeos en el Hospital Vasco de Quiroga en Morelia, Michoacán, en el que se identificaron 69 cepas, 41 provenientes de hongos filamentosos y 28 de levaduras. Los hongos más frecuentes fueron Candida, Penicillium y Aspergillus. En el presente trabajo, los hongos filamentosos más abundantes en lavados nasales y sedimentación por gravedad fueron Penicillium y Cladosporium, que se consideran parte de los agentes más comunes en la aparición de alergias respiratorias (Arenas 2008).

Durante el proceso de aerotransporte las partículas fúngicas pueden ser elevadas a la parte más alta de la capa de mezcla de la atmósfera, donde la viabilidad 


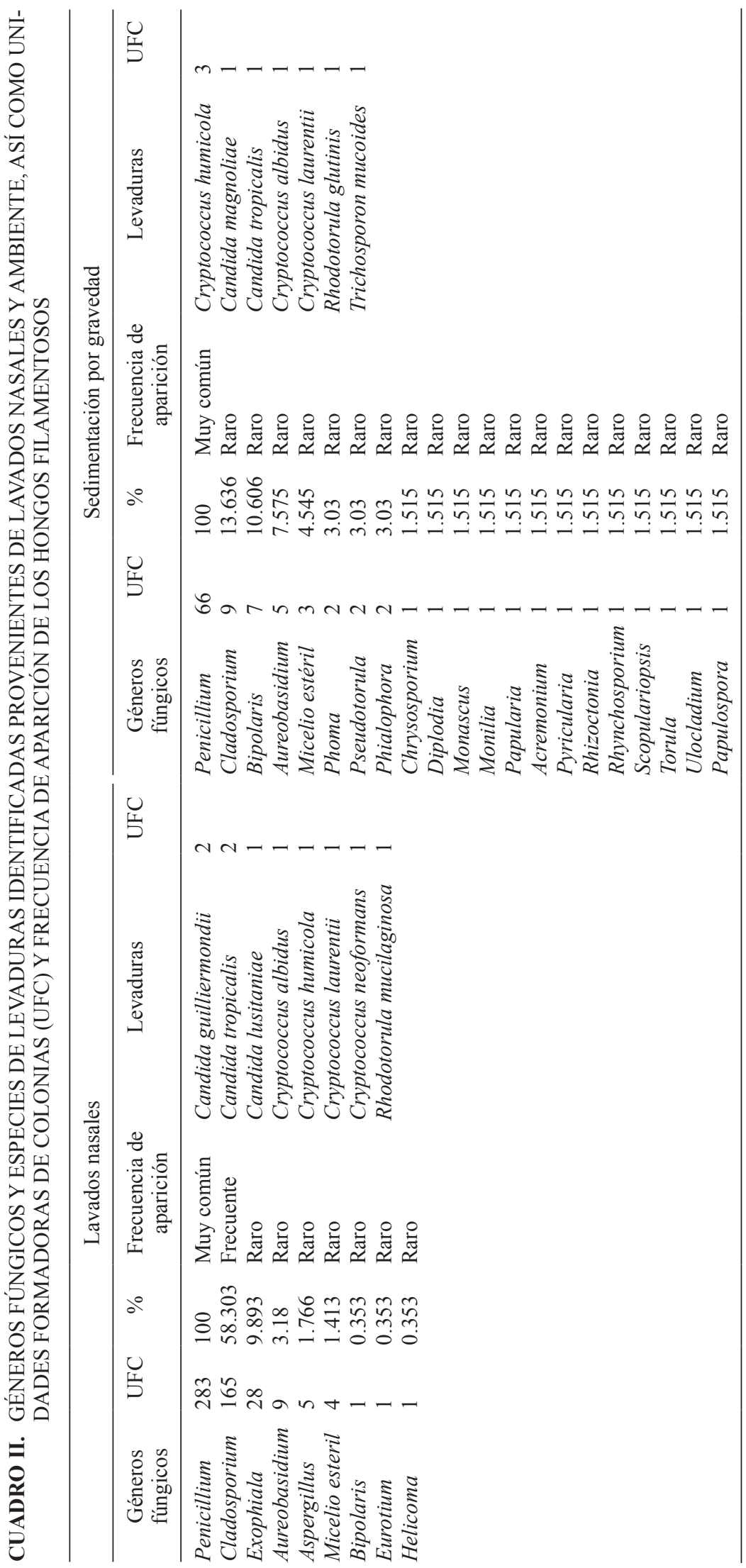


CUADRO III. CONTAMINANTES TOTALES OBTENIDOS DE LAVADOS NASALES OBSERVADOS EN MICROSCOPIO ÓPTICO DURANTE CADA UNO DE LOS MUESTREOS A LOS VOLUNTARIOS

\begin{tabular}{lcccc}
\hline \multicolumn{1}{c}{ Contaminante } & Primer lavado & \% de aparición & Segundo lavado & \% de aparición \\
\hline Epitelio & 228 & 100 & 250 & 100 \\
Fibras & 228 & 100 & 250 & 100 \\
Fragmentos de insectos & 95 & 41.666 & 100 & 40 \\
Conidios & 20 & 8.771 & 14 & 5.6 \\
Partículas de combustión & 50 & 21.929 & 42 & 4.8 \\
Fragmentos de micelio & 13 & 5.701 & 11 & 1.6 \\
Conidios con tubo germinal & 6 & 2.631 & 4 & 0 \\
Polen & 4 & 1.754 & 0 & 0 \\
Macroconidios Fusarium & 1 & 0.438 & 0 & 0.4 \\
Conidios Alternaria & 0 & 0 & 1 & 0.4 \\
Levaduras & 0 & 0 & 3 & 0 \\
Pelo & 0 & 0 & 0 & 1.6 \\
Fragmento vegetal & 4 & 1.754 & 4 & 0 \\
Conidios grupo Aspergillus/Penicillium & 2 & 0.8771 & 0 & 0 \\
Fragmentos de hifa & 5 & 2.192 & 250 & 0.8 \\
Bacterias & 228 & 100 & 0 & 2 \\
Esporas de pteridofita & 1 & 0.438 & 0 & 0.6 \\
Compuestos orgánicos & 0 & 0 & & 0 \\
\hline
\end{tabular}

A)
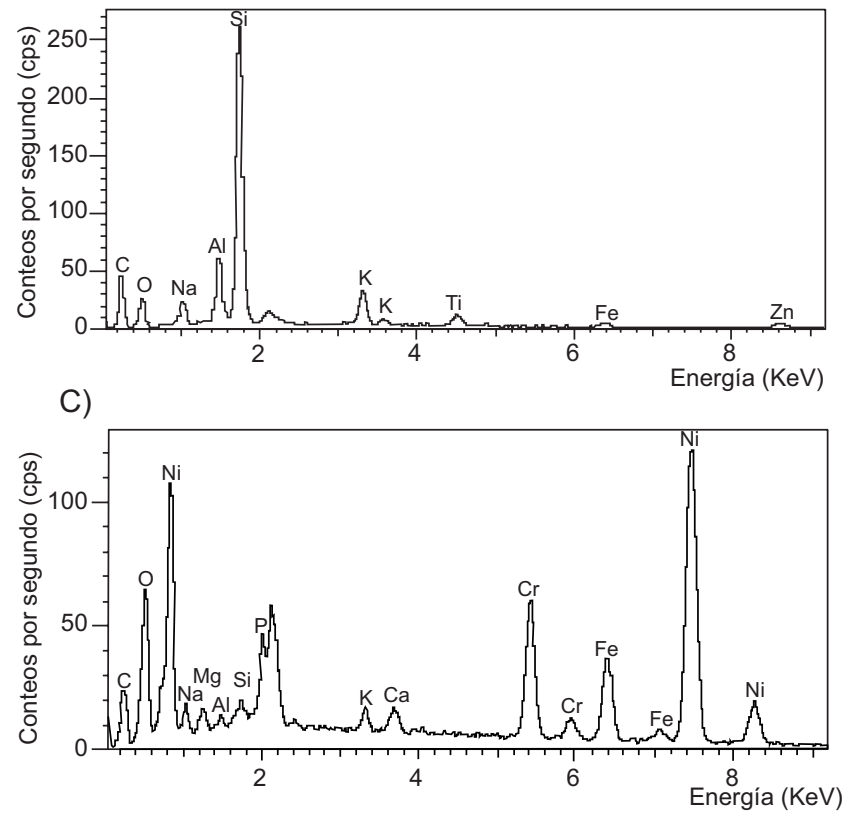

B)

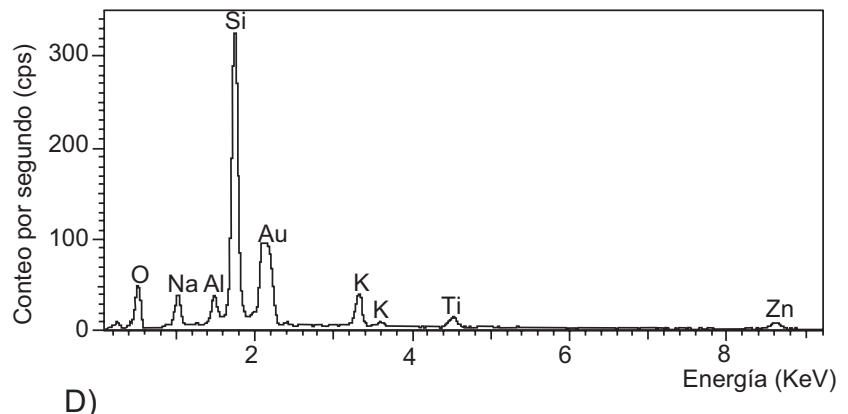

D)

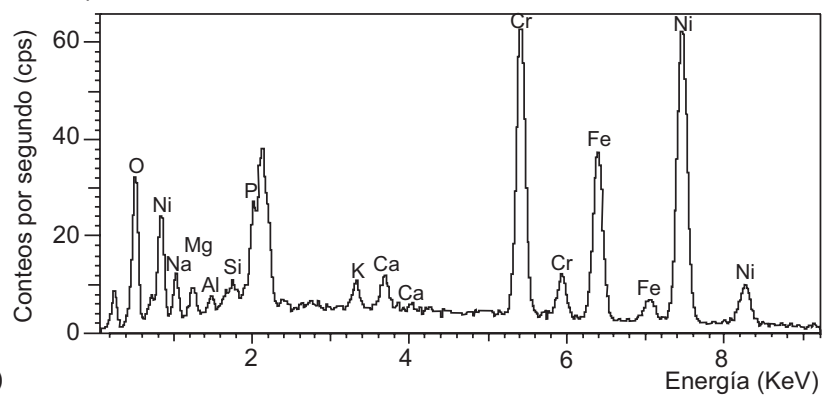

Fig. 1. Metales pesados determinados por análisis de química elemental de partículas presentes en muestras obtenidas por lavados nasales

de estos propágulos puede ser influenciada por factores meteorológicos como la radiación solar, la temperatura y la humedad relativa. La exposición a la radiación ultravioleta puede provocar inactivación o retraso en la germinación de los conidios debido a que causa desorganización de la membrana celular, desnatu- ralización proteica, estrés oxidativo y daño al ADN, ARN y ribosomas (Griffiths et al. 1998). Mientras que la temperatura en conjunto con la humedad relativa pueden desecar los propágulos (WMO 2010). Se debe resaltar el hecho de que la mayoría de los géneros identificados son clasificados como fitopatógenos, por lo 
que podrían estar presentes tanto en las áreas verdes de la universidad así como en distintos puntos de la ciudad por los que los voluntarios se transportaron antes de llegar al laboratorio para los lavados nasales. Al considerar el proceso de transporte aéreo de los propágulos fúngicos, así como los factores meteorológicos que pueden incidir en la inactivación de dichas partículas previo a la deposición en las vías respiratorias, se puede explicar en parte la falta de desarrollo micelial en la mitad de cajas de Petri sembradas con muestras de lavados nasales.

Se debe considerar que los bioaerosoles, aún sin ser viables, pueden tener implicaciones para la salud si presentan carga de toxinas o moléculas alergénicas como es el caso de las proteínas presentes en pared celular (Douwes et al. 2003, Huang et al. 2008, Weryszko-Chmielewska et al. 2017). Los síntomas del sistema respiratorio como respuesta a alérgenos fúngicos son más fuertes que los provocados por otros alérgenos presentes comúnmente en el ambiente. En parte, esto se debe a que los hongos tienen la capacidad de multiplicarse e infectar la piel o colonizar el tracto respiratorio (Krzysztofik 1992). Al ser inhaladas, las partículas fúngicas pueden desencadenar una de las reacciones más comunes, el asma, en la que los anticuerpos IgE están involucrados. El aumento en los cuadros de esta enfermedad es la razón por la que distintos investigadores buscan las causas de su formación, tanto genéticas como ambientales. Recientemente, se ha encontrado que la quitina, componente de la pared celular fúngica, provoca una fuerte respuesta inmune que puede llevar a alergias y asma alérgica (Toriello 2003, Lacey y West 2006, Reese et al. 2007, Van Dyken et al. 2011).

La identificación de levaduras permitió detectar once especies, pertenecientes a cuatro géneros. Las hongos levaduriformes pertenecientes al género Cryptococcus están distribuidos en todo el mundo y son frecuentemente aislados de suelo contaminado con heces de aves (Aberg y Powderly 1996, Rosario et al. 2005, Rosario et al. 2008), Las infecciones causadas por $C$. neoformans son las más comunes. Sin embargo, se ha observado el caso de infecciones emergentes con un incremento frecuente causadas por otras especies de Cryptococcus (Neves et al. 2015). Tal es el caso de C. laurentii y C. albidus, rara vez consideradas como patógenos de humanos (Lee et al. 2004, Burkin, 2007, Rodríguez y Pinilla 2012). La infección por Cryptococcus ocurre principalmente por la inhalación, a través del tracto alimenticio o por lesiones en la piel (Ellis y Pfeiffer 1990), los pulmones son clásicamente el sitio primario de infección (Lortholary et al. 2004).
Rhodotorula es un género compuesto por levaduras saprobias ubicuas, a las que se les ha reconocido como patógenos emergentes en humanos. Distintas especies se reconocen como comensales en la piel, las uñas y membranas mucosas, el tracto respiratorio superior y el digestivo, ya que aparecen en las heces (Pfaller et al. 2004, Richardson y Lass-Flörl 2008, Reyes et al. 2013). También pueden ser encontradas en productos lácteos, aire, agua, suelo, cortinas de baño y cepillos de dientes. Pueden contaminar el gel usado para el ultrasonido terapéutico, las soluciones intravenosas y los catéteres. $R$. mucilaginosa es la especie asociada de forma más frecuente a infecciones humanas (Murray et al. 2006, Reiss et al. 2011). Por otra parte, las levaduras del género Trichosporon se encentran comúnmente en el suelo y el agua. Además, este género pertenece a la flora normal de la piel humana y el tracto intestinal (Sageerabanoo et al. 2011, Capoor et al. 2015). T. mucoides ha sido reportada en casos severos de infecciones profundas en recién nacidos prematuros (Gökahmetogúlu et al. 2002, Silva et al. 2004) y en trasplantes de corazón, riñón e hígado (Nettles et al. 2003, Lacasse y Cleveland 2009).

Las infecciones por el género Candida son las micosis más frecuentes. Esta levadura forma parte de la microbiota del tracto respiratorio superior. Candida albicans es la mayor causa de candidiasis. Sin embargo, en las últimas décadas se ha comunicado de infecciones por especies distintas a C. albicans, principalmente candidemia e infecciones respiratorias asociadas a la colonización de catéteres venosos centrales, uso de antibióticos de amplio espectro y largas estancias intrahospitalarias, al aumento endémico del SIDA, incremento de la población madura, un mayor número de pacientes inmunocomprometidos y por el uso indistinto de materiales médicos (Silva et al. 2011, Martínez et al. 2014). C. guilliermondii raramente es aislada de pacientes como un patógeno y es relativamente menos estudiada que otras especies del género (Pasqualitto et al. 2006). Se puede aislar del suelo, plantas, insectos, agua de mar y de la atmósfera. Además es parte de la microbiota saprobia de la piel humana y de las superficies mucosas (Chien-Yuan et al. 2013). Por otra parte, C. lusitaniae es un agente etiológico infeccioso del tracto respiratorio y se reconoce como un patógeno oportunista nosocomial. Es un organismo único por su habilidad de desarrollar rápida resistencia durante el tratamiento con antifúngicos (Christenson et al. 1987, Hadfield et al. 1987). Así como estas levaduras identificadas durante la investigación pueden ser encontradas en distintos ambientes y conforman 
parte de la microbiota fúngica humana, su acción como patógenos oportunistas ocurre en condiciones de inmunosupresión u otros procesos debilitantes (Martínez et al. 2014) que pueden llevar a complicaciones mayores.

Al observar las muestras de lavados nasales en el microscopio óptico se identificaron distintas partículas de origen biológico. Así como contaminantes procedentes de la combustión.

Los metales pesados identificados por el análisis de química elemental fueron $\mathrm{Zn}, \mathrm{Cr}, \mathrm{Ni}, \mathrm{Fe}, \mathrm{Mg}$, $\mathrm{Ca}$ y $\mathrm{Cl}$ (Escobedo et al. 2001). Estos elementos, al ser parte de las partículas de la fracción respirable, representan un grave problema para la salud humana ya que aunque se puedan presentar en bajas concentraciones, la exposición constante representa complicaciones severas por su capacidad para irritar las fosas nasales y la garganta, inflamar los senos nasales, dañar la cavidad alveolar y aumentar el riesgo de padecer cáncer. También es necesario considerar las asociaciones de estos elementos en la atmósfera, ya que al provenir de distintas fuentes, la combinación para formar diversos compuestos representa un riesgo mayor (Prather et al. 2008).

Dependiendo del diámetro aerodinámico de las partículas inorgánicas que se encuentran suspendidas en el aire, estas pueden adherirse a los propágulos fúngicos, como se ha reportado en conidios del género Cladosporium (Weryszko-Chmielewska et al. 2017). Estas partículas pueden dañar la estructura de la pared de los conidios y de esta manera se facilita la entrada de compuestos químicos en el protoplasto; el impacto de estos contaminantes puede provocar alteraciones en las propiedades de las proteínas alergénicas (Ruffin et al. 1983, Nilsson 1990). Además, el daño mecánico producido a los conidios puede incrementar la liberación de sustancias alérgenas (Ghiani et al. 2012). Por otra parte, la Agencia para Sustancias Toxicas y el Registro de Enfermedades (ATSDR) y el Departamento de Salud y Servicios para Personas Mayores de Nueva Jersey (NJDHSS), mencionan la capacidad de los metales pesados para dañar las vías respiratorias superiores e inferiores, de esta manera se facilitan las infecciones fúngicas. Sin embargo, los factores de virulencia de los hongos son fundamentales para el desarrollo de enfermedades. Dentro de estos factores se encuentra la termotolerancia, crecimiento en medios como la sangre, resistencia a fagocitosis, mimetismo molecular (capacidad de un microorganismo patógeno de producir moléculas semejantes desde el punto de vista estructural, antigénico y funcional a otras moléculas del organismo anfitrión), excreción de enzimas, papel del hierro al ser cofactor de diversas metaloenzimas y adhesión (Murray et al. 2007, Arenas 2008).

\section{CONCLUSIÓN}

La implementación de los lavados nasales como estrategia de muestreo permitió obtener propágulos fúngicos viables cultivados en ARB, donde se identificaron distintos géneros de hongos filamentosos, entre ellos Penicillium y Cladosporium como los de mayor frecuencia de aparición. Esta técnica puede ser utilizada para conocer la microbiota fúngica nasal, además de poder ser de gran utilidad como parte de un diagnóstico de alergias respiratorias debido a la probabilidad de que la inhalación de estas partículas sea en lugares recurrentes de los afectados. Por otra parte, aunque se identificaron distintas levaduras que tienen potencial patógeno para el humano, la mayoría de ellas están reportadas como parte de la microbiota normal en el cuerpo, donde la probabilidad de una infección dependerá de distintos factores, entre ellos el estado del sistema inmunológico.

Como parte de las partículas presentes en los lavados nasales, se identificaron metales pesados por microscopía electrónica de barrido. Estos metales están presentes en el aire de la Ciudad de México y son producto de la combustión principalmente. Al ser inhalados pueden provocar cuadros alérgicos y en algunos casos daños físicos en distintos niveles del sistema respiratorio, donde pueden alcanzan a llegar a torrente sanguíneo y ser depositados en diversos órganos. Además, estos metales pueden tener un papel fundamental en la penetración de propágulos fúngicos en el organismo de manera que se facilita el proceso de infección.

En México existen distintos organismos gubernamentales como es la RAMA que se encarga del monitoreo de partículas inorgánicas presentes en el aire, dependiendo del diámetro aerodinámico y la cantidad en que son emitidas. Por otra parte, actualmente la Red Mexicana de Aerobiología (REMA) del Centro de Ciencias de la Atmósfera de la Universidad Nacioanl Autónoma de México, lleva a cabo el muestreo semanal de polen presente en el aire mediante la utilización de seis estaciones distribuidas en distintas alcaldías de la Ciudad. Sin embargo, para llevar a cabo una estrategia más adecuada de prevención ante el riesgo de afecciones respiratorias, es necesario que se lleve a cabo el monitoreo constante del total de bioaerosoles presentes en la atmósfera de la Ciudad de México. 


\section{REFERENCIAS}

Aberg J.A. y Powderly W.G. (1996). Cryptococcosis. Adv. Pharmacol. 37, 215-251. https://doi.org.10.1016/ S1054-3589(08)60951-5

Arenas R. (2008). Micología medica ilustrada. 5a ed. Mc Graw Hill. Ciudad de México, México, 448 pp.

Arx J.A. von. (1981). The genera of fungi sporulating in pure culture. Gartner Verlag, Berlín, Alemania, 424 pp.

Barnett H. L. y Hunter B. B. (1972). Illustrated genera of imperfect fungi 4 ed. Mc Millan. Nueva York, EUA, $218 \mathrm{pp}$.

Barron G.L. (1968). The genera of Hyphomycetes from soil. Robert Krieger Publish. Co. Florida, EUA, 364 pp.

Burkin C., Altintas N.D., Özkaya G., Serter S.S.T., Selcuk Z.T., Firat P., Arikan S., Cuenca-Estrella M. y Topeli A. (2007). Acute respiratory distress syndrome due to Cryptococcus albidus pneumonia: Case report and review of the literature. Med. Mycol. 45 (5), 469-473. https://doi.org.10.1080/13693780701386015

Bush R. K. y Portnoy J. M.. 2001. The role and abatement of fungal allergens in allergic disease. J. Allergy Clin. Immun. 107 (3), S430-S440. https://doi.org.10.1067/ mai.2001.113669

Capoor M.R., Agarwal S., Yadav S., Saxena A.K. y Ramesh V. (2015). Trichosporon mucoides causing onycomycosis in an immunocompetent patient. Int. J. Dermatol. 54, 704-7. https://doi.org.10.1111/ijd.12157

Chien-Yuan C., Shang-Yui H., Jih-Luh T., Woei T., Ming Y., Bo-Sheng K.C., Hwei-Fang T. y Po-Ren H. (2013). Clinical features of patients with infections caused by Candida guilliermondii and Candida fermentati and antifungal susceptibility of the isolates at a medical centre in Taiwan, 2001-10. J. Antimicrob. Chemoth. 68 (11), 2632-2635. https://doi.org.10.1093/jac/ dkt214

Christenson J.C., Guruswany A., Mukwaye G. y Rettig P.J. (1987). Candida lusitaniae: an emerging human pathogen. Pediatr. Infect. Dis. J. 6 (8), 755-757. https:// doi.org.10.1097/00006454-198708000-00013

Codina R., Fox R.W., Lockey R.F. y Bagg A. (2008). Typical levels of airborne fungal spores in houses without obvious moisture problems during a rainy season in Florida, USA. J. Investig. Allerg. Clin. 18 (3), 156-162.

Cooley J.D., Wong W.C., Jumper C.A. y Straus D.C. (1998). Correlation between the prevalence of certain fungi and sick building syndrome. Occup. Environ. Med. 55, 579-584. https://doi.org.10.1136/ oem.55.9.579

Cox S.C. y Wathes C.W. (1995). Bioaerosols handbook. CRC Press, Lewis Publishers, Boca Raton, Florida, EUA, 621 pp.
De la Rosa M.C., Mosso M.A. y Ullán C. (2002). El aire: hábitat y medio de transmisión de microorganismos. Observatorio Medioambiental 5, 375-402.

Denning D.W., Evans E.G.V., Kibbler C.C., Richardson M. D., Roberts M.M., Rogers T.R., Warnock D.W. y Warren R.E. (1997). Guidelines for the investigation of invasive fungal infections in haematological malignancy and solid organ transplantation. Eur. J. Clin. Microbiol. 16 (6), 424- 436. https://doi.org/10.1007/ BF02471906

Douwes J., Thorne P., Pearce N. y Heederik D. (2003). Bioaerosol health effects and exposure assessment: progress and prospects. Ann. Occup. Hyg. 47 (3), 187200. https://doi.org.10.1093/annhyg/meg032

Dutkiewicz J. (1997). Bacteria and fungi in organic dust as potential health hazard. Ann. Agr. Env. Med. 4, 11-16.

Ellis D.H. y Pfeiffer T.J. (1990). Ecology, life cycle, and infectious propagule of Cryptococcus neoformans. Lancet 336 (8720), 923-925. https://doi.org.10.1016/01406736(90)92283-N

Escobedo J.F., Victoria AR. y Ramírez A. (2001). La problemática ambiental en la ciudad de México generada por las fuentes fijas. Secretaría del Medio Ambiente [en línea]. http://www.bvsde.paho.org/bvsaidis/caliaire/ mexicona/r-0130.pdf 17/05/2017

Esquivel P., Mangiaterra M., Guisiano G. y Sosa M.A. (2003). Microhongos anemófilos en ambientes abiertos de dos ciudades del Nordeste argentino. Boletín Micológico 18, 21-28. https://doi.org.10.22370/bolmicol.2003.18.0.376

Ghiani A., Aina R., Asero R., Bellotto E. y Citterio S. (2012). Ragweed pollen collected along high-traffic roads shows a higher allergenicity than pollen sampled in vegetated areas. Allergy 67 (7), 887-894. https://doi. org.10.1111/j.1398-9995.2012.02846.x

Gioulekas D., Damialis A., Papakosta D., Spieksma F., Giouleka P. y Patalas D. (2004). Allergenic fungi spore records ( 15 years) and sensitization in patients with respiratory allergy in Thessaloniki, Greece. J. Invest Allerg. Clin. 14 (3), 225-231. https://doi. org.10.1027/0838-1925.16.2.52

Gökahmetogúlu S., Nedret A., Günes T. y Çetin N. (2002). Case reports. Trichosporon mucoides infection in three premature newborns. Mycoses 45 (3-4), 123-125. https://doi.org.10.1046/j.1439-0507.2002.00736.x

Griffiths H.R., Mistry P., Herbert K.E. y Lunec J. (1998). Molecular and cellular effects of ultraviolet lightinduced genotoxicity. Crit. Rev. Cl. Lab. Sci. 35 (3), 189-237. https://doi.org.10.1080/10408369891234192

Hadfield T.J., Smith M.B., Winn R.E., Rinaldi M.G. y Guerra C. (1987). Mycoses caused by Candida lusitaniae. Rev. Infect. Dis. 9 (5), 1006-1012. https:// doi.org.10.1093/clinids/9.5.1006 
Hafidh M., Harney M., Kane R., Donnelly M., Landers R. $y$ Smyth D. (2007). The role of fungi in the etiology of chronic rhinosinusitis: A prospective study. Auris Nasus Larynx 34 (2), 185-189. https://doi.org.10.1016/j. anl.2006.07.007

Hernández-Castillo O., Mugica-Álvarez V., CastañedaBriones M., Murcia J., García F. y Falcón Y. (2014). Aerobiological study in the Mexico City subway system, Aerobiologia 30 (4), 357-367. https://doi. org.10.1007/s10453-014-9334-6

Hinds W.C. (1999). Aerosol technology: Properties, behavior, and measurement of airborne particles. $2 \mathrm{a}$ ed. Wiley-Interscience Publication. Los Angeles, California, EUA, 504 pp.

Huang R., Agranovski I., Pyankov O. y Grinshpun S. (2008). Removal of viable bioaerosol particles with a low- efficiency HVAC filter enhanced by continuous emission of unipolar air ions. Indoor Air 18 (2), 106112. https://doi.org.10.1111/j.1600-0668.2007.00512.x

Kacker S.K. (2010). Looking after local nasal and sinus mucosa in health, disease and after surgery. Indian J. Otolaryngol. 62 (3), 258-263. https://doi.org.10.1007/ s12070-010-0085-6

Krzysztofik B. (1992). Air microbiology [Polish]; Warsaw: Wyd. Politechniki. Varsovia, Polonia, 198 pp.

Lacasse A. y Cleveland K.O. (2008). Trichosporon mucoides fungemia in a liver transplant recipient: a case report and review. Transpl. Infect. Dis. 11 (2), 155-159. https://doi.org.10.1111/j.1399-3062.2008.00355.x

Lacey M.E. y West J.S. (2006). The air spora. A manual for catching and indentifying airborne biological particles. Spring. Dordrecht, Holanda, 156 pp. https://doi. org.10.1007/978-0-387-30253-9

Lee Y.A., Kim H.J., Lee T.W., Kim M.J., Lee M.H., Lee J.H. e Ihm Ch. G. (2004). First report of Cryptococcus albidus - Induced disseminated cryptococcosis in a renal transplant recipient. Korean J. Intern. Med. 19 (1), 53-57. https://doi.org. 10.3904/kjim.2004.19.1.53

Lortholary O., Nunez H., Brauner M.W., y Dromer F. (2004). Pulmonary cryptococcosis. Semin. Resp. Crit. Care M. 25 (2), 145-157. https://doi. org.10.1055/s-2004-824899

Martínez I.M., González M. y Torres H.K. 2014. Identificación molecular de Candida lusitaniae en infección de tracto respiratorio inferior. Rev. Argent. Microbiol. 46 (4), 307-310. https://doi.org.10.1016/S03257541(14)70087-2

Murray P.R., Rosenthal K.S. y Pfaüer M.A. (2007). Microbiología médica. 5a ed. Elsevier. Madrid, España, 890 pp.

Nettles R.E., Nichols L.S., Bell-McGuinn K., Pipeling M.R., Scheel P.J.Jr. y Merz W.G. (2003). Successful treatment of Trichosporon mucoides infection with fluconazole in a heart and kidney transplant recipient.
Clin. Infect. Dis. 15, 36 (4), E63-6. https://doi.org. 10.1086/367665

Neves R.P., Lima-Neto R.G., Leite M.C., Silva V.K., Santos F. de A. y Macêdo D.P. (2015). Cryptococcus laurentii fungaemia in a cervical cáncer patient. Bra. J. Infect. Dis. 19 (6), 660-663. https://doi.org.10.1016/j. bjid.2015.06.014

Nilsson S. (1990). Regional and global distribution of aeroallergens. Rev. Palaeobot. Palyno. 64 (1-4), 2934. https://doi.org.10.1016/0034-6667(90)90113-W

Oliveira M., Riberio H., Delgado L., Fonseca J., CastelBranco M.G. y Abreu I. (2010). Outdoor allergenic fungal spores: Comparison between an urban and a rural area in Northern Portugal. J. Investig. Allergol. Clin. Immunol. 20 (2), 117-128.

OMS (2005). Manual de Bioseguridad en el laboratorio. 3a ed. Organización Mundial de la Salud. Ginebra, Suiza, 210 pp.

Pasqualitto A.C., Ventura A.G. y Severo L.C. (2006). Candida guilliermondii as the aetiology of candidosis. Rev. Inst. Med. Trop SP. 48 (3), 123-127. https://doi. org.10.1590/S0036-46652006000300002

Pfaller M.A. y Diekema D.J. (2004). Rare and emerging opportunistic fungal pathogens: concern for resistance beyond Candida albicans and Aspergillus fumigatus. J. Clin. Microbiol. 42 (10), 4419-4431. https://doi. org.10.1128/JCM.42.10.4419-4431.2004

Ponce-Caballero C., Cerón-Palma I., López-Pacheco M., Gamboa-Marrufano M. y Quintal-Franco C. (2010). Indoor-outdoor fungal-aerosols ratios of domestic homes in Merida, Mexico. Ingeniería 14 (3), 169-175.

Prather K.A., Hatch C.D. y Grassian V.H. (2008). Analysis of atmospheric aerosols. Annu. Rev. Anal. Chem. 1, 485-514. https://doi.org.10.1146/annurev. anchem.1.031207.113030

Ramírez E. 2000. Estudio para la expansión de la Red Automática de Monitoreo Atmosférico de la Ciudad de México y Zona Conurbada. [en línea]. http:// www.bvsde.paho.org/bvsaidis/mexico11/ca-9.pdf $17 / 05 / 2017$

Reese T.A., Liang H.E., Tager A.M., Luster A.D., Van Rooijen N., Voehringer D. y Locksley R.M. (2007). Chitin induces accumulation in tissue of innate immune cells associated with allergy. Nature 447 (7140), 92-96. https://doi.org.10.1038/nature05746

Reiss E., Shadomy H.J. y Lyon M.G. (2011). Fundamental medical mycology. Wiley-Blackwell. Nueva Jersey, EUA, 656 pp. https://doi.org.10.1002/9781118101773

Reponen T., Grinshpun A., Conwell K., Wiest J. y Anderson M. (2001). Aerodynamic versus physical size of spores: measurement and implication on respiratory deposition. Grana. 40 (3), 119-125. https://doi. org. $10.1080 / 00173130152625851$ 
Reyes I., Pérez L., Morffi M. y Barletta J.E. (2013). Aislamiento de Rhodotorula. Presentación de un caso en paciente con leucemia mieloide aguda. Medisur 11 (5), 542-545.

Richardson M. y Lass-Flörl C. (2008). Changing epidemiology of systemic fungal infections. Clin. Microbiol. Infec. 5-24. https://doi.org.10.1111/j.14690691.2008.01978.x

Rocha A., Alvarado M.A., Gutierrez R., Salcedo, S.M. y Moreno S. (2013). Variación temporal de esporas de Alternaria, Cladosporim, Coprinus, Curvularia y Venturia en el aire del área Metropolitana de Monterrey, Nuevo León, México. Rev. Int. Contam. Ambie. 29 (2), 155-165.

Rodríguez D.A. y Pinilla A.P. (2012). Infección asociada a catéter central por Cryptococcus laurentii en niño críticamente enfermo: a propósito de un caso y revisión del tema. Infectio 16 (1), 72-74. https://doi.org.10.1016/ S0123-9392(12)70059-0

Rodríguez-Orozco A.R., Vargas E., Tafolla L., Ruíz H., Hernández L.A. y Vázquez S. (2008). Géneros fúngicos aislados de pacientes con rinitis alérgica y su relación con la prueba de hipersensibilidad subcutánea de Prick. Rev. Mex. Micol. 28, 89-94.

Rosario I., de Mendoza M.H., Déniz S., Soro G., Alamo I. y Acosta B. (2005) Isolation of Cryptoccocus species including C. neoformans from cloaca of pigeons. Mycoses. 48 (6), 421-424. https://doi.org.10.1111/j.14390507.2005.01153.x

Rosario I., Acosta B. y Colom F. (2008). La paloma y otras aves como reservorio de Cryptococcus spp. Rev. Iberoam. Micol. 25 (1), S13-S18. https://doi. org.10.1016/S1130-1406(08)70020-2

Rosas I., Calderón C., Gutiérrez S. y Mosiño P. (1986). Airborne fungi isolated from rain water collected in Mexico City. Rev. Int. Contam. Ambie. 2 (1), 13-23.

Rosas I., Escamilla B., Calderón C. y Mosiño P. (1990). The daily variations of airborne fungal spores. Aerobiologia 6, 153. https://doi.org.10.1007/BF02539108

Rosas I., Calderón C., Martínez L., Ulloa M. y Lacey J. (1997). Indoor and outdoor airborne fungal propagule concentrations in Mexico City. Aerobiologia 13 (1), 23-30. https://doi.org.10.1007/BF02694787

Rosique J., Fócil R. y Cid A. (2013). Hongos del aire de una zona suburbana de la ciudad de Villahermosa, Tabasco. Kuxulkab'19 (37), 23-27.

Ruffin J., Williams D., Banerjee U. y Pinnix K. (1983). The effects of some environmental gaseous pollutants on pollen-wall proteins of certain airborne pollen grains. Grana. 22 (3), 171-175. https://doi. org.10.1080/00173138309427703

Sáenz C. y Gutiérrez M. (2003). Esporas atmosféricas en la comunidad de Madrid. Instituto de Salud Pública. 5a ed. Comunidad de Madrid, Consejería de Sanidad. Madrid, España, 49 pp.

Sageerabanoo, Malini A., Oudeacoumar P. y Udayashankar C. (2011). Onychomycosis due Trichosporon mucoides. Indian J. Dermatol Ve., 77 (1), 76-7. https://doi. org.10.4103/0378-6323.75001

SEMARNAT (2002). Norma Oficial Mexicana NOM087-SSA1. Protección ambiental-Salud ambiental-Residuos peligrosos biológico-infecciosos-Clasificación y especificaciones de manejo. Secretaría de Medio Ambiente y Recursos Diario Oficial de la Federación. 17 de febrero de 2003.

SEMARNAT (2011). Programa para mejorar la calidad del aire de la Zona Metropolitana del Valle de México 2011-2020. Gobierno del Estado de México. Toluca, México, 388 pp.

Sercombe J.K., Green B.J. y Tovey E.R. (2006). Recovery of germinating fungal conidia from the nasal cavity after environmental exposure. Aerobiologia 22 (4), 295-304. https://doi.org/10.1007/s10453006-9043-x

Silva S., Díaz M.C. y Febré N. (2004). Invasive fungal infections in Chile: a multicenter study of fungal prevalence and susceptibility during a 1 -year period. Med. Mycol. 42 (4), 333-339. https://doi.org.10.1080/ 13693780410001657153

Silva S., Negri M., Henriques M., Olveira R., Williams D.W. y Azeredo J. (2011). Candida glabrata, Candida parapsilosis and Candida tropicalis: biology epidemiology, pathogenicity and antifungal resistance. FEMS Microbiol. Rev. 36 (2), 288-305. https://doi. org.10.1111/j.1574-6976.2011.00278.x

Torres-Rodríguez J.M., Pulido-Marrero Z. y Vera-García Y. (2012). Respiratory allergy to fungi in Barcelona, Spain; Clinical aspects, diagnosis and specific treatment in a general allergy unit. Allergol. Immunopath. 40 (5), 295-300. https://doi.org.10.1016/j. aller.2011.09.003

Toriello C. (2003). Alergias por hongos. En: Microbiología y parasitología médica. (Z.J. Tay, Q.M. Gutiérrez, M.R. López, L. J. Molina, y M. E. Manjarrez, Eds.). 3a ed. Méndez Cervantes Editores, México D.F., México, $1134 \mathrm{pp}$.

Van Dyken S.J., García D., Porter P., Huang X., Quinlan P.J., Blanc P.D., Corry D.B. y Locksley R.M. (2011). Fungal chitin from asthma- associated home environments induces eosinophilic lung infiltration. J. Immunol. 187 (5), 2261-2267. https://doi.org.10.4049/ jimmunol.1100972

Verhoeff A.P. y Burge H.A. (1997). Health risk assessment of fungi in home environments. Ann. Allerg. Asthma Im. 78 (6), 544-556. https://doi.org.10.1016/S10811206(10)63214-0 
Weryszko-Chmielewska E., Kasprzyk I., Nowak M., Sulborska A., Kaczmarek J., Szymanska A., Haratym W., Gilski M. y Jedryczka M. (2017). Health hazards related to conidia of Cladosporium - biological air pollutants in Poland, central Europe. J. Environ. Sci-China 65, 271-281. https://doi.org.10.1016/j.jes.2017.02.018

WMO (2010). Aerobiology. En: Guide to agricultural meteorological practices. World Meteorological Organization. Ginebra, Suiza, 794 pp.

WMA (1964). Declaration of Helsinki-ethical principles for medical research involving human subjects. World Medical Association [en linea]. https://www.wma.net/ es/policies-post/declaracion-de-helsinki-de-la-ammprincipios-eticos-para-las-investigaciones-medicasen-seres-humanos/ 16/06/2020

Wu P.C., Tsai J.C., Li F.C., Lung S.C. y Su H.J. (2004). Increased levels of ambient fungal spores in Taiwan are associated with dust events from China. Atmos. Environ. 38 (29), 4879-4886. https://doi.org.10.1016/j. atmosenv.2004.05.039

Yeo H. y Kim J. (2002). SPM and fungal spores in the ambient air of west Korea during the Asian dust (yellow sand) period. Atmos. Environ. 36 (35), 5437-5442. https://doi.org.10.1016/S1352-2310(02)00672-6 